\title{
Entre orthodoxie positiviste et histoire universitaire
}

La controverse Aulard-Robinet autour de Danton

Between Positivist Orthodoxy and Academic History: the Controversy between Aulard and Robinet on the subject of Danton

\section{Alric Mabire}

\section{OpenEdition}

\section{Journals}

\section{Édition électronique}

URL : https://journals.openedition.org/ahrf/12928

DOI : $10.4000 /$ ahrf.12928

ISSN : 1952-403X

Éditeur :

Armand Colin, Société des études robespierristes

Édition imprimée

Date de publication : 1 décembre 2013

Pagination : $3-23$

ISBN : 978290832789

ISSN : 0003-4436

\section{Référence électronique}

Alric Mabire, "Entre orthodoxie positiviste et histoire universitaire », Annales historiques de la Révolution française [En ligne], 374 | octobre-décembre 2013, mis en ligne le 01 décembre 2016, consulté le 01 juillet 2021. URL : http://journals.openedition.org/ahrf/12928; DOI : https://doi.org/10.4000/ahrf. 12928 


\title{
ENTRE ORTHODOXIE POSITIVISTE ET HISTOIRE UNIVERSITAIRE : LA CONTROVERSE AULARD-ROBINET AUTOUR DE DANTON
}

Alric MABIRE

\begin{abstract}
Alphonse Aulard est souvent présenté comme un dantoniste forcené face à Albert Mathiez, fondateur des AHRF. Cet article revient sur une polémique autour de Danton, opposant en 1893 Alphonse Aulard au docteur Robinet, un disciple d'Auguste Comte. La controverse permet d'analyser les actes politiques de Danton au prisme de la philosophie de l'Histoire positiviste et de la notion de « dictature », afin de questionner la République et la formation de l'histoire révolutionnaire comme histoire méthodique.
\end{abstract}

Mots-clés : Danton, républicanisme, positivisme, école méthodique, historiographie, Alphonse Aulard, Auguste Comte, docteur Robinet.

La commémoration du Bicentenaire de la Révolution française a permis, dans les années 1980, de renouveler l'intérêt pour la Révolution française auprès d'un large public. Dans ce cadre, Danton, un personnage assez oublié depuis l'avant-guerre, a fait sa réapparition au travers d'un film (Wajda, 1982) et de plusieurs publications ${ }^{1}$ qui corrigèrent le regard parfois caricatural de Wajda. Ce fut l'occasion d'apprécier la vitalité de

(1) Frédéric Bluche, Danton, Paris, Perrin, 1984 ; Daniel Lacotte, Danton, le tribun de la Révolution, Paris, P. M. Favre, 1987 ; Jan BASzKIEWICZ, Danton, Warszawa, Instytut Wydawiczy, 1980 ; Norman HAMPSON, Danton, Oxford, Blackwell, 1988 ; Plus récemment, se reporter à Anne SIMONIN, «Qui a tué Georges Danton? », dans Pascal MorVAN (dir.), Mélanges en l'honneur de Yves Guchet, Bruxelles, Bruylant, 2008, p. 613-642. 
la légende dantonienne, actualisée par les données de la Guerre froide ${ }^{2}$. De leur côté, les universitaires, « considérant que Mathiez et ses disciples avaient clos le dossier, se sont désintéressés de Danton $»^{3}$. Plus récemment, le centenaire de la Société des études Robespierristes ${ }^{4}$, ainsi que l'actualité des manuscrits de Robespierre ont renforcé la visibilité de celui-ci aux dépens de son adversaire. Le propos de cet article est de reprendre le dossier Danton où Albert Mathiez l'a laissé. Car dans la bataille que ce dernier a menée pour légitimer son entreprise historiographique, il lui a fallu déconstruire la position dantoniste, « officielle », qu'Alphonse Aulard incarnait alors ${ }^{5}$. En 1927, lors d'une conférence au Grand Orient de France, Mathiez l'exprimait très clairement :

«Il s'agit de savoir si les politiques \& les publicistes qui, en 1887 \& en 1891, ont élevé une statue à Danton à Arcis-sur-Aube, son pays, d'abord, à Paris ensuite, si ces hommes, qui ont prétendu réhabiliter le grand corrompu, un siècle après son supplice, ont eu raison contre la Convention nationale unanime, contre le Tribunal révolutionnaire unanime, contre les contemporains unanimes, contre tous les Républicains de la première moitié du XIX ${ }^{\mathrm{e}}$ siècle unanimes $»^{6}$.

Mathiez entendait « [renouer] la tradition $»^{7}$ concernant la probité de Danton, contre ce groupe de «politiques \& publicistes » composés d'Alphonse Aulard, de Jules Clarétie, d'Antonin Dubost et du docteur Robinet $^{8}$. Derrière cette liste, ce qui était visé, c'était la Société de l'histoire de la Révolution et sa revue La Révolution française que tous avaient fréquentée ou fréquentaient encore.

Il est évident que pour les besoins de la querelle, Albert Mathiez a eu tendance à simplifier les positions des uns et des autres, notamment celles de son ancien maître, Aulard. Et c'est sur cette base que se sont fondées la SER et les $A H R F$. Or, en 1893, Danton avait déjà été le sujet d'une controverse, au sein même de la revue La Révolution française et de

(2) Voir Michel VovelLe, « Sur le " Danton" de Wajda : la Révolution n'est pas un "délire" », dans Combats pour la Révolution française, Paris, La Découverte, 2001, p. 341-347.

(3) Frédéric BLUCHE, op. cit., p. 10.

(4) Voir $A H R F, n^{\circ} 353,2008$.

(5) Voir James FRIGUGLIETTI, Albert Mathiez, historien révolutionnaire (1874-1932), Paris, SER, 1974, notamment le chapitre 3 qui s'intéresse au robespierrisme d'Albert Mathiez. Plus récemment, James FRIGUGLIETTI, «La querelle Mathiez-Aulard et les origines de la Société des études robespierristes », $A H R F, \mathrm{n}^{\circ} 353,2008$, p. 63-94.

(6) Albert MATHIEZ, « Danton. L'histoire et la légende », dans Girondins et Montagnards, Paris, Éditions de la Passion, 1988, p. 260-261 (1 éd., Paris, Firmin Didot \& Cie, 1930).

(7) Id., Autour de Danton, Paris, Payot, 1926, p. 9.

(8) Id., « Danton. L'histoire et la légende », op. cit., p. 261. 
la Société de l'histoire de la Révolution'. S'étaient alors affrontés le tenant d'une tradition positiviste orthodoxe, le docteur Robinet, et le titulaire de la récente chaire d'Histoire de la Révolution française, Alphonse Aulard. Deux interprétations qui, malgré leurs dantonismes respectifs, ne s'accordaient pas sur le sens historique à donner aux actions du révolutionnaire.

L'étude des controverses s'est beaucoup développée ces dernières années, notamment dans les Science Studies et en histoire intellectuelle, plus attentive aux réseaux et contextes, aux argumentaires et aux stratégies de légitimation des uns et des autres. Elle montre que les disciplines scientifiques sont toujours en formation et que la divergence d'opinion, loin de freiner la recherche, contribue au contraire à la développer et à renforcer sa fonction socialisatrice ${ }^{10}$. Cette polémique, qui préfigure celle qu'initiera Mathiez contre Aulard, témoigne avec force de la complexité épistémologique de cette fin du $\mathrm{XIX}^{\mathrm{e}}$ siècle, alors que se rationalise la méthode de l'histoire comme discipline scientifique ${ }^{11}$.

Deux angles d'approches sont ainsi envisagés. D'abord, cette controverse, l'une parmi tant d'autres du champ historiographique révolutionnaire $^{12}$, pose la question de la manière dont elle se développe : la rupture entre Aulard et le docteur Robinet empêche ce dernier de répondre légitimement dans La Révolution française. Ce faisant, elle définit par l'exclusion une frontière entre le monde positiviste et militant et celui de l'université et des revues d'histoire et donne à voir la construction d'une histoire méthodique de la Révolution française telle que pratiquée par Alphonse Aulard $^{13}$. Ensuite, parce que le conflit marque la coupure entre deux systèmes historiques, une attention particulière sera portée sur l'interprétation

(9) Cette controverse est très succinctement mentionnée dans l'ouvrage de Claude NICOLET, L'idée républicaine en France (1789-1924), Gallimard, 1994 (1éd. 1982), p. 241.

(10) Voir Mil neuf cent. Revue d'histoire intellectuelle, $\mathrm{n}^{\circ} 25,2007 / 1$, notamment Jean-Louis FABIANI, « Disputes, polémiques et controverses dans les mondes intellectuels. Vers une sociologie historique des formes de débat agonistique », p. 45-60. Voir également Raisons politiques, $\mathrm{n}^{\circ} 47$, $2012 / 3$.

(11) Christian DelacroIX, François DossE et Patrick GaRCIA, Les courants historiques en France, Paris, A. Colin, 1999, p. 80-85.

(12) Voir James FrigugLieTti, « La querelle Mathiez-Aulard et les origines de la Société des études robespierristes », art. cit. et Julien LOUVRIER, «Penser la controverse : la réception du livre de François Furet et Denis Richet, La Révolution française », AHRF, n³51, 2008/1, p. 151-176.

(13) Sur l'ensemble théorique et pratique de cette légitimation, voir le programme ambitieux qu'Alphonse Aulard défend lors de sa leçon d'ouverture du cours à la Sorbonne (Alphonse AULARD, «Histoire de la Révolution française », La Révolution française (désormais indiquée $R F$ ), Tome X, 1886/1, p. 868-897, reprise dans Alphonse AULARD, Études et leçons sur la Révolution française, première série, Paris, F. Alcan, 1893, p. 3-40). Sur le contexte qui entoure la formation du cours, puis sa transformation en chaire d'histoire de la Révolution française, voir Claudine WoliKow, « Centenaire dans le Bicentenaire : 1891-1991. Aulard et la transformation du cours en chaire d'Histoire de la Révolution française à la Sorbonne », $A H R F, \mathrm{n}^{\circ} 286,1991$, p. 431-458. 
de la place de Danton en tant qu'acteur de l'histoire, et sur la notion de « dictature » qu'il aurait exercée.

\section{Cadre et enjeux de la controverse}

La revue La Révolution française a longtemps été associée, à juste titre, au nom d'Alphonse Aulard, qui la dirige de 1887 à sa mort en 1928. Sous sa direction, elle opéra un profond changement de ligne éditoriale, pour devenir une revue historique à part entière, affiliée à l'école méthodique en formation ${ }^{14}$. Cependant, lorsqu'elle est fondée en 1881 par Auguste Dide, un publiciste républicain, La Révolution française est une revue militante. Aux côtés de Dide se trouvent Jean-Claude Colfavru, ancien révolutionnaire de 1848, ainsi qu'Étienne Charavay, un archivistepaléographe qui apporte une caution scientifique à l'entreprise éditoriale. Leur objectif est d'engager un mouvement unitaire autour de la célébration du Centenaire de la Révolution française ${ }^{15}$. Pour y répondre, la revue se fait l'écho d'initiatives locales et publie des articles historiques destinés à mieux faire connaître la période révolutionnaire et ses acteurs.

De fait, Danton, " plus calomnié que connu ${ }^{16}$, est très tôt le sujet d'une série d'études en particulier celles du docteur Robinet ${ }^{17}$. Celui-ci est un disciple convaincu d'Auguste Comte ${ }^{18}$, dont il a été l'un des treize exécuteurs testamentaires. Véritable institution, l'Exécution Testamentaire avait pour tâche « d'assurer la publication de [l'œuvre d'Auguste Comte], l'extension de la doctrine et la conservation de ses archives de la rue Monsieur le Prince ${ }^{19}$. Robinet avait choisi de s'occuper de Danton. Ses

(14) Voir Olivier LÉVY-Dumoulin, « Revues historiques », dans Christian DELACROIX, François Dosse, Patrick GARCIA et Nicolas OFFEnSTADT (dir.), Historiographies, concepts et débats, Paris, Gallimard, 2010, p. 587-588.

(15) Sur le rôle joué par Auguste Dide dans la préparation du Centenaire de la Révolution, voir Raymond HUARD, « Le Centenaire de 1789 et les origines du parti radical, la Fédération de 1889 », dans Le XIX ${ }^{e}$ siècle et la Révolution française : journées d'études de Nanterre, octobre 1989, Paris, Créaphis, 1992,p. 129-146.

(16) Maurice SPRONCK, $R F$, Tome I, 1881, p. 442.

(17) Jean-François Eugène Robinet, médecin puis conservateur-adjoint de la section du musée Carnavalet affectée à Paris, a déjà publié deux études consacrées à Danton lorsqu'il commence à écrire dans La Révolution française : Danton. Mémoire sur sa vie privée, Paris, Chamerot et Lauwereyns, 1865 et Le procès des Dantonistes, Paris, E. Leroux, 1879.

(18) «Le docteur Robinet adhéra complètement, en effet, à la plupart des innovations religieuses préconisées par Auguste Comte, spécialement à la conception théorique et pratique des sacrements positivistes » (Émile CORRA, Lettres d'Auguste Comte au docteur Robinet, Paris, Société Positiviste Internationale, 1926, p. 8).

(19) Alice GÉRARD, «Les disciples «complets » de Comte et la politique positive (1870-1914) », dans Annie PETIT (dir.), Auguste Comte. Trajectoires positivistes, 1789-1998, Paris, L'Harmattan, 2003, p. 287. 
interventions dans La Révolution française sont nombreuses : en 1881, il soutient le projet d'élévation d'une statue de Danton, qui n'aboutit que dix ans plus tard ${ }^{20}$; ensuite, c'est par une réédition sous forme d'articles de son Danton. Mémoire sur sa vie privée que le docteur apporte sa plus importante contribution ${ }^{21}$; en 1888 et 1889, La Révolution française publie in extenso des chapitres tirés de Danton. Homme d'État qui est publié à la fin $1889^{22}$.

De son côté, Alphonse Aulard est lui aussi un contributeur précoce de la revue, dans laquelle il écrit dès 1881 . Normalien, agrégé et auteur d'une thèse sur Léopardi en 1878, Aulard débute ses études révolutionnaires en s'intéressant à l'éloquence parlementaire pendant la Révolution ${ }^{23}$. Il consacre un article à Danton en 1882 et reconnaît sa sympathie pour les auteurs positivistes ${ }^{24}$. Par ailleurs, il signe, à partir de 1884, sous le pseudonyme Santhonax, une tribune intitulée «Les Lundis révolutionnaires » dans le journal de Clemenceau La Justice. L'omniprésence du tribun comme sujet d'études dans les premières années de La Révolution française coïncide avec l'ambition politique qui la porte. Lorsque Aulard écrit que «l'action était presque tout Danton ${ }^{25}$, il fait écho au livre d'Antonin Dubost, Danton et la politique contemporaine ${ }^{26}$. Les premiers numéros de la revue sont consensuels à propos du tribun, considéré comme un homme politique d'exception.

Les années 1880 sont marquées par un profond changement universitaire qui, par l'introduction des cours fermés en $1883^{27}$ et la segmentation des périodes historiques ${ }^{28}$, permet l'émergence de spécialistes aux dépens

(20) Docteur RoBINET, « La statue de Danton », $R F$, Tome I, 1881, p. 478-483.

(21) Ces articles seront la base d'une troisième édition de l'ouvrage chez Charavay (Docteur RoBinet, Danton. Mémoire sur sa vie privée, Paris, Charavay Frères, 1884).

(22) Docteur RoBINET, Danton Homme d'État, Paris, Charavay Frères, 1889.

(23) Claudine WOLIKOW, art. cit., p. 437.

(24) Alphonse AULARD, «Études sur l'éloquence de Danton », RF, 1882/2, p. 118. Il écrit en 1884 une biographie qu'il renie quelques années plus tard, car trop inspirée par les Positivistes (Alphonse AUlARD, Danton, Paris, Picard-Bernheim, 1884).

(25) Alphonse AULARD, «Études sur l'éloquence de Danton », art. cit. , p. 115

(26) Antonin Dubost, Danton et la politique contemporaine, Versailles, Cerf et fils, 1877. Dubost a participé à La Révolution française en donnant une série d'articles sur Danton et les massacres de septembre (voir Antonin DUBOST, « Danton et les massacres de septembre », RF, Tome VII, 1884/2, p. 166-170, 193-218, 352-359, 385-405, 511-533), et il a fait partie du comité directeur de la Société de l'histoire de la Révolution. Plus modéré que le docteur Robinet, il est proche de Littré (voir Claude NICOLET, L'idée républicaine en France..., op. cit., p. 239-241).

(27) Ces cours sont destinés à un public homogène d'étudiants, considérés désormais comme tels et favorisant le développement d'un discours scientifique (voir Christian DELACROIX, François Dosse et Patrick GARCIA, Les courants historiques en France, op. cit., p. 70)

(28) Claudine WolIKOW, art. cit., p. 436. 
des généralistes. La nomination de Louis Liard comme directeur de l'Enseignement supérieur entre 1884 et 1902 engage un mouvement d'autonomisation administrative des universités qui se caractérise par l'accroissement des cours et des chaires d'histoire en Sorbonne ${ }^{29}$. C'est pourquoi l'approche du Centenaire pousse plusieurs municipalités de tendance radicale à ouvrir des enseignements sur la Révolution française ${ }^{30}$. Alphonse Aulard, du fait de ses amitiés politiques, est le candidat idéal pour le cours d'histoire de la Révolution française qui ouvre à la Sorbonne ${ }^{31}$. Dès sa leçon inaugurale, il se place en historien et annonce son programme : « Notre ambition sera moins de juger que de faire connaître. [...] La légende a recouvert cette période de notre histoire d'incrustations dont la plupart sont encore intactes : nous tâcherons de les arracher et de vous mettre en présence de la réalité nette et nue $»^{32}$.

L'importance prise par Aulard dans le champ des études révolutionnaires et son ambition scientifique expliquent sans doute le choix d'Auguste Dide de le nommer à la tête de La Révolution française qui se scinde en deux dès $1887^{33}$. C'est sur cette base que le nouveau directeur-rédacteur en chef entend développer son projet, puisque désormais la revue «n'admettra que des études originales, composées d'après les sources $»^{34}$. Ce mouvement se concrétise en 1888 dans la formation de la Société de l'histoire de la Révolution - dont Aulard est secrétaire-général avant même que les statuts soient arrêtés - qui prend la suite du Comité d'études pour la préparation historique du Centenaire de 1789. La revue devient l'organe officiel de cette société en $1890^{35}$.

Parallèlement, avec le concours de l'État (commissions ministérielle et municipale), Aulard entreprend l'édition de nombreuses sources : Recueil des actes du Comité de salut public et Procès-verbaux de la Société des Jacobins à partir de 1889. Enfin, en 1892, le cours d'histoire de la Révolution française est transformé en chaire ${ }^{36}$. Le docteur Robinet n'est pas absent

(29) Christian DELACROIX, François DosSE et Patrick GARCIA, Les courants historiques en France, op. cit., p. 73.

(30) À Paris en 1886, à Lyon en 1887 et à Toulouse en 1889.

(31) Claudine WOLIKOW, art. cit., p. 442.

(32) Alphonse AUlARD, «Leçon d'ouverture du cours sur la Révolution française », $R F$, Tome X, 1886/1, p. 879 .

(33) S'opère une distinction entre revue militante et revue historique. Auguste Dide dirige la revue militante, chargée d'organiser les comités du Centenaire. Le projet, transformé en Fédération 89 , fut sans lendemain. (voir Raymond HUARD, art. cit.).

(34) $R F$, Tome XII, $1887 / 1$, p. 577

(35) Ibidem, Tome XIV, 1881/1, p. 852-854 et Tome XVIII, 1890/1, p. 481.

(36) Claudine WoliKOW, art. cit., p. 452-455. 
de ce mouvement puisqu'il est nommé vice-président de la Société de l'histoire de la Révolution française à partir de $1890^{37}$.

Ouvrant l'année universitaire 1892-1893, Aulard annonce : «Messieurs, je me propose d'étudier cette année la vie et la politique de Danton $»^{38}$. La leçon inaugurale qu'il donne alors est toute consacrée à Auguste Comte et sa conception de la philosophie de l'Histoire ${ }^{39}$. Il s'y montre très critique, parfois virulent, contre les «formules religieuses » des positivistes. Publiée dans La Révolution française et dans la Revue Bleue entre décembre 1892 et janvier 1893, cette leçon conduisit à la démission du docteur Robinet de la Société de l'histoire de la Révolution qui s'expliqua en ces termes :

« Ne pouvant en aucune façon accepter et subir l'exécution que M. le professeur Aulard a bien voulu faire de ma personne et de mes modestes recherches historiques, en Sorbonne, dans son discours d'ouverture, j'ai pensé qu'il valait mieux me retirer de notre Société, que d'y risquer des contacts et des discussions pour le moins désagréables $»^{40}$.

Bien sûr, Aulard s'est toujours défié d'avoir voulu «exécuter» qui que ce soit, mais sa prééminence au sein de la Société et de $L a$ Révolution française empêchait qu'une controverse raisonnable s'établisse par ces organes, quoique le comité qui reçut la demande de démission l'eût fortement encouragée. La controverse se déporta donc vers d'autres terrains, dans la Revue Occidentale ${ }^{41}$, organe des positivistes orthodoxes, où Émile Antoine défendit la « théorie positive » de la Révolution française, ainsi que dans des pamphlets anonymes ${ }^{42}$.

Pourtant, ces publications ne firent l'objet que d'une seule mention dans le numéro du 14 mai 1893 de La Révolution française. Le docteur Robinet, identifié comme l'auteur des écrits anonymes, fut déconsidéré parce que sa réponse « [n'était] pas polie » et que « son esprit [n'était] pas

\footnotetext{
(39) «C'est à Auguste Comte lui-même, et non à ses disciples, qu'il faut demander la théorie positiviste sur l'histoire de la Révolution » (Ibid., p. 10).

(40) $R F, 1893 / 1$, p. 179.

(41) Émile ANTOINE, «La théorie positive de la Révolution française », Revue Occidentale, 1893 , p. $253-290$.

(42) Aulard rend compte de ces publications mais souligne que le docteur Robinet « n'admettrait jamais qu'Auguste Comte ait pu se tromper en rien, [et que] son esprit n'est pas libre » $(R F$, 1893/1, p. 475).
}

(38) Alphonse AULARD, "Auguste Comte et la Révolution française », $R F$, Tome XXIV, $1893 / 1$, p. 5 
libre $»^{43}$. En revanche, Aulard reconnut qu'Émile Antoine avait répliqué avec « beaucoup d'ingéniosité et de courtoisie ${ }^{44}$ quoique cette courtoisie fût gênée par des attaques personnelles : « On me reproche d'être encombrant, d'écrire dans trop de Revues [...]. C'est vrai : j'écris beaucoup trop. [...] Mais que ces querelles personnelles ou anonymes sont fastidieuses ! Retournons au travail, à la science, à la vérité $»^{45}$. La controverse, telle que la pratique Aulard à la Sorbonne et dans sa revue, est sensible à la temporalité. Alphonse Aulard se positionne en juge et critique a posteriori de la formulation des doctrines positivistes, et cela renforce son point de vue pour deux raisons : la première, c'est qu'il se montre en action, en train de faire de l'histoire - car il ne se contente pas de critiquer Comte et ses disciples, il reprend le dossier Danton, et en donne une nouvelle interprétation ; la seconde, c'est que ce dialogue diachronique légitime l'école méthodique comme école scientifique, dépolitisée, née sous la IIIe République, c'est-à-dire dans un environnement propice au développement des sciences ${ }^{46}$.

A l'inverse, le docteur Robinet, en répondant par des pamphlets anonymes à son contradicteur, est discrédité parce qu'il utilise des moyens désuets. Désormais, les controverses historiques doivent se faire dans les revues d'histoire, pas en dehors. Par ailleurs, elles doivent se faire dans la «courtoisie » et le respect de l'autre, élément que les contradicteurs d'Aulard ne respectent pas. C'est pourquoi la controverse Aulard / Robinet définit un champ cohérent de la recherche scientifique, seul capable d'accueillir les divergences d'opinion. Elle est le support de l'auto-légitimation du groupe en formation autour du maitre de la Sorbonne, mouvement de professionnalisation de l'historien, avec ses revues, ses sociétés, et ses pratiques de la divergence scientifique.

En ce sens, ce qui est surtout reproché aux Positivistes orthodoxes, en particulier au docteur Robinet, c'est leur incapacité à mettre à distance travail théorique et prise de position politique. Les débats sur l'inauguration de la statue de Danton à Paris en 1891 en attestent. Dès 1881, La Révolution française avait soutenu le projet d'élévation d'une statue à la gloire du tribun. En 1888, la Société de l'Histoire de la Révolution française, à travers la figure du docteur Robinet ${ }^{47}$, assistait à l'inauguration de la statue de

\footnotetext{
(43) $R F$, Tome XXIV, 1893/1, p. 475.

(44) Ibidem.

(45) Ibid., Tome XXIV, 1893/1, p. 476

(46) Christian DelaCroIX, François Dosse et Patrick GARCIA, Les courants historiques en France, op. cit., p. 67.

(47) Qui est aussi le représentant de la Société positiviste de Paris.
} 
Danton d'Arcis-sur-Aube ${ }^{48}$. Or, en 1891, à Paris, elle n'est pas présente. Claudine Wolikow a avancé l'idée que cette absence se justifiait par «le refus d'Aulard de céder à l'utilitarisme philosophique ou politique qui « défigure » l'histoire ${ }^{49}$. On trouve dans La Révolution française une autre explication : «Cette abstention n'avait certes rien de volontaire. Nous n'avions pas été invités à cette cérémonie et voilà pourquoi nul orateur n'a parlé en notre nom ${ }^{50}$. Que l'absence soit volontaire ou non, elle confirme bien la mise à distance de la portée politique du travail historique ${ }^{51}$. Par ailleurs, Aulard avait vivement contesté l'introduction, au Sénat, d'une polémique à propos de Danton : «Ces paperasses d'Archives, si aimables dans la paisible salle de travail de la rue des Francs-Bourgeois, faisaient une figure bien indiscrètement désagréable à la tribune d'une assemblée politique $\gg^{52}$. Il y a donc un temps, un lieu et des manières de pratiquer la controverse. Et c'est précisément tout le programme d'Aulard que de donner à l'histoire de la Révolution française les mêmes assises que celles de l'école méthodique pour les autres périodes ${ }^{53}$, en s'interdisant de polémiquer autrement que dans un champ scientifique reconnu comme tel ${ }^{54}$.

C'est autour « de la conception du caractère et du rôle $»^{55}$ du tribun révolutionnaire que se développe l'argumentaire aulardien au profit d'une nouvelle manière de faire de l'histoire. Deux nœuds orientent la critique : la philosophie de l'histoire d'Auguste Comte d'une part et le concept de « dictature » d'autre part ${ }^{56}$.

(48) $R F$, Tome XV, $1888 / 2$, p. 369-381

(49) Claudine WOLIKOW, art. cit., p. 456.

(50) Alphonse AULARD, «L'inauguration de la statue de Danton », RF, Tome XXI, 1891/2, p. 97.

(51) Christian DelacroiX, François DossE et Patrick GARCIA, Les courants historiques en France, op. cit., p. 95

(52) Alphonse AULARD, «L'inauguration de la statue de Danton », art. cit., p. 98

(53) Voir Patrick GARCIA, « Historiographie méthodique », dans Christian DELACROIX, François Dosse, Patrick GARCIA et Nicolas OFFEnSTADT, Historiographies, concepts et débats, op. cit., p. 443-452 et Claudine WolIKOW, art. cit., p. 450.

(54) Du moins pour ce qui concerne purement la discipline historique. Aulard n'hésite pas, en d'autres occasions, à s'investir dans la cité et à prendre position. Voir Alphonse AULARD, Polémique et Histoire, Paris, E. Cornély, 1904.

(55) Alphonse AULARD, «Auguste Comte et la Révolution française », art. cit., p. 9.

(56) Aulard ne revient absolument pas sur la « vie privée » de Danton. Il est intimement convaincu de la probité du tribun (« Il n'y a plus moyen de dire aujourd'hui que Danton était vénal, sanguinaire, ignorant, fanatique. On le dit tout de même encore, mais on le dit comme pamphlétaire et non comme historien », Ibid.). 


\section{Danton dans le mouvement de l'Histoire}

Le docteur Robinet, nous l'avons dit, est un disciple orthodoxe du positivisme. Fidèle aux conceptions de son maître, il appréhende le passé comme un développement complexe mais linéaire, qui explique le présent et peut annoncer l'avenir. Chaque époque déterminée par le système comtien s'insère dans une continuité générale ${ }^{57}$. Chez Robinet, cette marche du progrès est clairement énoncée :

« Elle nous montre l'élite de notre espèce guidant ses masses immenses, depuis l'origine des premières associations humaines, pour élever progressivement l'homme de l'isolement et de la bestialité primitifs, à l'état de perfectionnement qui doit caractériser un jour la sociabilité finale $»^{58}$.

Dans ses cours, Auguste Comte a identifié trois états généraux de l'évolution de l'humanité : l'état "primitivement théologique », l'état « transitoirement métaphysique » qui culmine dans la Révolution française, et l'état « finalement positif » caractérisant la société finale, celle qu'appellent les Positivistes ${ }^{59}$. Le passage d'un état à l'autre est un processus lent et progressif, auquel le fondateur du positivisme est très attentif. Ainsi, chaque moment historique est toujours la matrice de « deux mouvements simultanés de décomposition politique [aussi appelé mouvement négatif] et de recomposition sociale [soit mouvement positif] $»^{60}$, ces deux mouvements s'articulant entre eux pour permettre la marche du progrès.

Ainsi, deux éléments moteurs conduisent le mouvement de l'histoire chez les Positivistes : d'un côté le facteur humain, «l'élite [qui guide les] masses immenses »; de l'autre les facteurs intellectuels (mouvement négatif / mouvement positif) qui entrainent l'ensemble de la société humaine ${ }^{61}$. Dans ce schéma, la Révolution française marque l'aboutissement de la « Révolution occidentale » qui commence au XIV ${ }^{\mathrm{e}}$ siècle, portée par un

(57) Bien qu'inspiré directement de Condorcet, Auguste Comte s'en démarque par un « esprit historique et relativiste : toutes les époques ont eu leur raison d'être et méritent un " intérêt sympathique" » (Alice GÉRARD, « La Révolution française d'Auguste Comte », dans Cristina CASSINA (dir.), Sociologica, politica e religione : la filosofia di Comte per il diciannovesimo secolo, Pise, Edizioni Plus, 2001, p. 69).

(58) Docteur RoBINET, Notice sur l'œuvre et la vie d'Auguste Comte, Paris, Dunod, 1860, p.

(59) Auguste ComTE, Cours de philosophie positive. Tome IV, Paris, Bachelier, 1839, p. 653.

(60) Id., Cours... Tome VI, Paris, Bachelier, 1842, p. 344.

(61) Id., Cours... Tome IV, op. cit., p. 648. 
mouvement négatif contre l'état théologique, et occupe une place spécifique, moins par son exceptionnalité que par la réduction qu'elle opère sur des forces historiques anciennes.

Dans la thèse comtienne, trois moments et écoles philosophiques traversent la Révolution française, dont deux négatifs - les écoles de Voltaire et de Rousseau - et une positive - l'école de Diderot. Chacune d'elle aurait régné successivement sur la Révolution ${ }^{62}$. Comme pour les Positivistes, il est impossible de « concevoir une action politique indépendante d'une action philosophique ${ }^{63}$, Danton est associé à l'école de Diderot, seule capable d'organiser la société ${ }^{4}$. La construction historiographique du docteur Robinet est de faire coïncider les actions politiques de Danton avec le système légué par le maître du positivisme. Trois moments fondamentaux sont distingués :

$1 \%$ Danton contribue à mettre fin à la monarchie (mouvement négatif).

$2 \%$ Danton organise le système républicain (mouvement positif).

$3 \%$ Danton prépare la défense nationale (mouvement positif) ${ }^{65}$.

Ce programme, vaste et ambitieux, ne peut être envisagé qu'à la condition que soit prouvée la pleine conscience historique de l'acteur, qui le pousse à l'avant-garde de ses contemporains ${ }^{66}$. C'est la raison pour laquelle Robinet entend convaincre ses lecteurs du républicanisme précoce de son sujet, ce, afin de prouver que la philosophie de l'histoire de Comte fonctionne, et que Danton est le « principal fondateur de l'ordre républicain $»^{67}$. Reste à savoir de quel « ordre républicain » il est question.

(62) L'école de Voltaire règne huit mois, celle de Diderot dix mois et celle de Rousseau quatre mois. Comme l'écrit Alice Gérard, « Le schéma de la Révolution française est aussi beaucoup plus clair en devenant ternaire : chacun des moments-clés (1789, 1793, 1794 avant Thermidor) correspond à une des trois écoles (Voltaire, Diderot, Rousseau), et chacune de celle-ci a l'ascendant relatif d'un des trois états (métaphysique, positif, théologique), dans un désordre révélateur de la marche difficile du progrès » (Alice GÉRARD, « La Révolution française d'Auguste Comte », art. cit., p. 84).

(63) Ibid., p. 83.

(64) Dans sa leçon d'ouverture, Aulard est très critique à l'égard de ce schéma. «Comme c'est systématique ! », s'exclame-t-il, avant de montrer qu' «il est insoutenable de présenter comme de purs négateurs, comme de purs destructeurs [les philosophes qui ont édifié la base du droit nouveau] et c'est de la fantaisie de dire qu' au XVIII ${ }^{\mathrm{e}}$ siècle, en dehors de Diderot, il n'y a eu que négativisme » (Alphonse AUlARD, « Auguste Comte et la Révolution française », art. cit., p. 18-19).

(65) Docteur RoBINET, « Danton, d'après les documents », RF, Tome III, 1882/2, p. 387.

(66) Cette interprétation fait d'ailleurs écho au rôle que s'attribuent eux-mêmes les disciples orthodoxes de Comte, que leur petit nombre renforce dans la conviction « que ce sont les avant-gardes, organes du mouvement social, qui font l'histoire, et non pas les masses, inertes et faillibles » (Alice GÉRARD, «Les disciples "complets" d'Auguste Comte... », art. cit., p. 288).

(67) Docteur RoBInET, Danton Homme d'État, op. cit., p. 9. 
Dès son plus jeune âge, écrit Robinet, Danton aurait rencontré la «manne intellectuelle $»^{68}$ du républicanisme antique, partagée par de nombreux penseurs du XVIII ${ }^{\mathrm{e}}$ siècle, qu'il dépasse par une lecture assidue d'auteurs modernes. Ainsi, l'inventaire de sa bibliothèque révèle un « indice de cet instinct politique supérieur qui le portait à étudier surtout la civilisation contemporaine, et qui l'éloignait de toute imitation pédante du passé ${ }^{69}$. Alphonse Aulard ne dit guère davantage lorsqu'il considère que la « nouveauté » de Danton réside avant tout dans sa rhétorique ${ }^{70}$. Mais cette nouveauté permet à Robinet d'associer le révolutionnaire au courant physiocratique et aux idées de Turgot dont le programme politique, relu par lui, ne serait au fond qu'une organisation politique centralisée par «un roi ou un président de la République $\gg^{71}$ exerçant les fonctions exécutives et législatives. En conséquence, le républicanisme prêté à Danton est un républicanisme de circonstance, car la République dépend de l'homme d'État qui la gouverne : doit-il être un roi, alors elle sera monarchiste. Les Positivistes orthodoxes, à l'instar d'Auguste Comte, étaient très réticents à l'égard de la démocratie. Aulard le souligne fortement dans ses critiques. Mais la particularité de la période révolutionnaire, c'est que celui qui aurait dû engager la marche du progrès, à savoir Louis XVI, n'avait pas la conscience historique suffisante pour le faire, et il fallut lui trouver un substitut dans la personne de Danton, dictateur républicain ${ }^{72}$.

Chez Robinet, la perspicacité de Danton et son opportunisme politique sont le signe d'une prescience de ce qui est en train de se jouer et du rôle qu'il doit y mener. Le tribun était « disponible quand se leva l'aurore de 1789. Matériellement et moralement, il était prêt ; prêt aussi intellectuellement $\gg^{73}$. À l'inverse, toute l'opération historiographique d'Alphonse Aulard vise à déconstruire cette position d'avant-garde. Lorsqu'il reprend le dossier, et qu'il étudie les traces des actions du révolutionnaire, Aulard

(68) Id., « Les commencements de Danton », RF, Tome XV, 1888/2, p. 102.

(69) Id., «Danton, d'après les documents », $R F$, Tome IV, 1883/1, p. 581-582. L'utilisation de l'expression «pédante » rappelle les critiques d'Auguste Comte à l'égard de la pédantocratie, c'est-à-dire du monde des savants. Ce terme lui avait été soufflé par John Stuart Mill.

(70) «Danton rejetait les règles de la rhétorique traditionnelle. Ses harangues ne sont ni composées, ni écrites comme celle des anciens ou même de Mirabeau et de Robespierre » (Alphonse AULARD, «Études sur l'éloquence de Danton », art. cit., p. 194). Il semble que l'illusion sur les lectures « modernes » du tribun ait subsisté longtemps (voir Frédéric BLUCHE, Danton, op. cit., p. 26).

(71) Souligné par nous. Docteur RoBINET, Danton Homme d'État, op. cit., p. 35.

(72) Claude NICOLET, L'idée républicaine en France..., op. cit., p. 240. Sur la « dictature », voir infra. La problématique de la conscience historique est primordiale. Comte explique que, faute d'une théorie suffisante de la loi du progrès qu'il découvre en 1822, les hommes de la Révolution, en dehors de Condorcet et de Danton ne pouvaient « raisonner que de façon "anti-historique" » (Alice GÉRARD, « La Révolution française d'Auguste Comte », art. cit., p. 71).

(73) Docteur RoBINET, «Les commencements de Danton », RF, Tome XV, 1888/2, p. 120 
refuse, par manque de preuve, de conclure à son républicanisme ${ }^{74}$. Au mieux, écrit-il, « [ses biographes positivistes] ont pris pour une profession de foi républicaine les cris de colère qu'il poussa avec le peuple, à la nouvelle de la fuite du roi $»^{75}$. Ce que relève l'étude, c'est avant tout le pragmatisme de l'homme politique, ses hésitations et la mise en perspective de son rôle au regard de celui des autres personnalités de la Révolution. C'est pourquoi, conclut Aulard, c'est une « erreur que de le présenter comme le fondateur du parti républicain en France, comme le premier qui ait voulu la République, qui l'ait demandée. [...] Il hésite, il ne croit pas l'heure venue, [...] il suit prudemment l'opinion $»^{76}$.

Dans les deux biographies, l'analyse de Danton est très différente : elle est sous-tendue par des conceptions divergentes de l'action politique. Pour Robinet, le grand homme explique le mouvement révolutionnaire. Il surplombe et guide la masse d'un peuple comparé à des « agglomérations enfiévrées qui, susceptibles parfois d'une irrésistible et salutaire exaltation, ne sont soumis, par cela même, à aucune règle, à aucune discipline sérieuse, et ne peuvent en aucun cas, être pris pour des organes permanents et normaux de direction ${ }^{77}$. Chez Aulard, de tendance radicale-socialiste, le travail historique se fait au contraire beaucoup plus minutieux, pour retrouver, dans l'histoire, le mouvement de l'opinion et de la multiplicité des acteurs historiques ${ }^{78}$.

C'est pourquoi le rôle historique - et historiographique - de Danton dépend étroitement des capacités d'agir que l'historien lui accorde et donc, en quelque sorte, de l'acceptation ou non de son statut de dictateur/ homme d'État.

\section{Danton dans les journées révolutionnaires}

En 1873, le docteur Robinet publiait une courte notice pour répondre au journal de Gambetta, La République française, qui avait critiqué son ouvrage Danton. Mémoire sur sa vie privée. Le nœud de la controverse se

(74) Alphonse AUlaRD, « Danton au district des Cordeliers et à la Commune », $R F$, Tome XXIV, 1893/1, p. 114-115.

(75) Id., « Danton en 1791 et en $1792 », R F$, Tome XXIV, 1893/1, p. 313.

(76) Ibidem, p. 322

(77) Docteur RoBinet, Danton Homme d'État, op. cit., p. 53.

(78) C'est tout le programme du livre d'Alphonse AULARD, Histoire politique de la Révolution française. Origines et développement de la démocratie et de la République (1789-1804), Paris, A. Colin, 1901. Sur cet ouvrage et son interprétation, voir Jacques GODECHOT, Un jury pour la Révolution, Paris, R. Laffont, 1974, p. 231-282 et sa discussion chez Claude NICOLET, L'idée républicaine en France, op. cit., p. 97-101. 
situait autour de l'interprétation à donner au 10 août 1792. Pour Robinet, il était évident que Danton était l' " âme du mouvement du 10 août » ${ }^{79}$, parce que cela correspondait d'une part au programme que les Positivistes lui avaient assigné ${ }^{80}$, d'autre part parce qu'il était impossible, dans la théorie de Comte, de penser la spontanéité d'un événement historique ${ }^{81}$. Pour traduire le 10 août, Robinet employait le concept de force :

«L'on entend par force, en sociologie, le concours d'un nombre variable, mais toujours à peu près défini, ou au moins assignable, de personnes ou de groupes de personnes vers un même but intellectuel ou politique, résumé par un individu $»^{82}$.

Chez Robinet, ce concept est toujours employé pour justifier la primauté de Danton sur les journées révolutionnaires.

Voici comment est justifiée sa paternité sur le 10 août : Danton aurait préparé la journée depuis « que la possibilité de renverser le trône et de proclamer la République lui [a] été démontrée ${ }^{83}$, en s'entendant d'abord avec les chefs des Fédérés venus à Paris en juin pour organiser une force militaire ; dans la soirée du 9 au 10 août, il aurait poussé toutes les sections à nommer les commissaires qui allaient former la Commune insurrectionnelle, et cela grâce à ses « intermédiaires »; c'est encore lui qui pousserait la Commune de Paris, dans laquelle il a des « intelligences », à abdiquer au profit de la Commune insurrectionnelle ; enfin, il aurait arrêté Mandat, le chef de la Garde nationale, dicté son arrêt de mort et l'aurait remplacé par Santerre ${ }^{84}$. Par ailleurs, sa retraite d'Arcis-sur-Aube, du 6 au 9 août 1792 trahirait l'assurance de la réussite de son coup ${ }^{85}$. La démonstration implique du lecteur qu'il associe le club des Cordeliers et les « intermédiaires » à Danton lui-même ${ }^{86}$. Cette lecture des faits s'explique par un problème théorique à résoudre : comment parler de Danton en dictateur avant son

(79) Docteur RoBINET, « Danton d'après les documents », $R F$, Tome III, 1882/2, p. 389.

(80) Voir supra.

(81) Comme le souligne Alice Gérard, il n’y a aucune évocation, chez Comte, « des fameuses "journées", chères à Michelet » (Alice GÉrARD, « La Révolution française d'Auguste Comte », art. cit., p. 75).

(82) Docteur RoBinet, Le Dix Août et la symbolique positiviste, Paris, E. Leroux, 1873, p. 6.

(83) Id., Danton Homme d'État, op. cit., p. 96. Démonstration pour le moins bancale puisque les seuls indices qui permettent d'en juger sont la destitution des ministres girondins, le 14 juin, et la lettre virulente de La Fayette à l'Assemblée législative, dénonçant les clubs et les partis, le 16 juin.

(84) Cette arrestation est considérée comme le fait décisif de la journée (Ibidem, p. 96).

(85) Ibid., p. 94-98. Nous ne discuterons pas la véracité de ces affirmations. Pour une analyse récente et documentée du rôle de Danton lors du 10 août, voir Frédéric BLUCHE, Danton, op. cit., p. $173-178$.

(86) Robinet explique cependant que Danton était mieux placé, lors du 10 août, en tant que procureur de la Commune de Paris, pour diriger le mouvement révolutionnaire que s'il avait été député 
entrée au gouvernement central ? Robinet n'emploie jamais directement le mot de «dictature » lorsqu'il parle des journées révolutionnaires, et c'est pourquoi il passe par les notions floues d'influence, d'intermédiaire et d'ascendance morale pour expliquer comment Danton résume seul l'ensemble du mouvement révolutionnaire.

Chez Aulard, le portrait de Danton est beaucoup plus mitigé. C'est avant tout un agitateur local qui ne possède aucune influence en dehors de son district, puis de sa section. Il joue un rôle obscur lors du 14 juillet $1789^{87}$, est peu visible les 5 et 6 octobre ${ }^{88}$, n'est pas l'auteur de la pétition des Cordeliers du 17 juillet $1791^{89}$. Son potentiel ne se mesure qu'à son art oratoire et sa capacité à toucher un public de plus en plus large ${ }^{90}$. Lorsqu'il agit, il n'est jamais seul. Ainsi, la retraite d'Arcis-sur-Aube est présentée comme le signe qu'il n'est pas l'unique organisateur de la journée du 10 août ${ }^{91}$, ce qui amène Alphonse Aulard à considérer que " sans lui, le trône n'en aurait pas moins été renversé à cette époque. Mais il est permis de croire que, si Danton n'avait pas mis son énergie et son esprit pratique au service de cette révolution, elle eût été plus violente, plus hasardeuse, plus sanglante $\gg^{92}$.

Homme prépondérant ou acteur parmi d'autre, chaque analyse a sa propre version du rôle de Danton dans les surgissements des journées révolutionnaires. En fin de compte, la version d'Aulard vise à faire admettre qu' «il n'y a que les coups d'État rétrogrades qui puissent être considérés comme l'œuvre d'un seul homme : les révolutions dans le sens du progrès sont plutôt anonymes, je veux dire nationales ${ }^{93}$. Manière

ou ministre, « car il faisait partie [...] du gouvernement même de Paris » (Docteur RoBINET, Le Dix Août et la symbolique positiviste, op. cit., p. 9).

(87) Alphonse AUlARD, «Danton au district des Cordeliers et à la Commune », art. cit., p. 116.

(88) Ibid., p. 118

(89) Chez Robinet, le 17 juillet 1791 est présenté comme la préfiguration ratée du 10 août 1792 (Docteur RoBINET, «Danton et le club des Cordeliers en 1791 », RF, Tome XVII, 1889/2, p. 145). Aulard démontre que Danton n'est jamais venu au Champ de Mars et qu'il n'est pas l'auteur de la pétition des Cordeliers (Alphonse AULARD, «Danton en 1791 et en 1792 », art. cit., p. 318).

90) « La tribune cordelière n'avait pour auditoire que Paris, c'était à la tribune des Jacobins que l'on parlait à toute la France. [...] Ce n'était que là que Danton pouvait se faire connaître du pays, se transformer, d'agitateur parisien qu'il était, en conseiller de l'opinion française, et jouer le rôle d'un Mirabeau ou d'un Robespierre » (Alphonse AULARD, « Danton au club des Cordeliers et au département de Paris », RF, Tome XXIV, 1893/1, p. 240).

(91) Id., « Danton et la Révolution du 10 août 1792 », RF, Tome XXIV, 1893/1, p. 391.

(92) Ibidem, p. 397

(93) Ibid., p. 385. 
habile de reprendre les termes positivistes pour les décharger de leur sens universaliste au profit d'une histoire certes linéaire, mais surtout nationale ${ }^{94}$.

\section{Danton dictateur ?}

Nous avons vu quelles étaient les interprétations divergentes concernant le républicanisme de Danton et son implication dans les journées révolutionnaires. Il reste à déterminer ce que signifie la prise effective du pouvoir par Danton, c'est-à-dire interroger la notion de « dictature » dans les écrits du docteur Robinet.

La dictature est centrale et problématique dans la philosophie positiviste ${ }^{95}$. Cette notion qui vient du système romain et s'actualise par la Révolution française ${ }^{96}$ est reprise par Auguste Comte lorsqu'il commence à écrire sous la Restauration. Pour lui, la « crise » est une donnée fondamentale du contemporain ${ }^{97}$, et l'évocation de la figure du dictateur relève d'une double valeur performative : elle affirme une continuité entre le passé et le présent, tout en répondant au besoin d'organiser la société future. De ce fait, la sociologie comtienne n'est pas éloignée du contexte dans lequel elle est pensée ${ }^{98}$, ce qui signifie qu'elle doit s'adapter à l'époque dans laquelle elle se trouve.

La notion de dictature, telle que l'a léguée le maître à ses disciples, est à la fois la force motrice envisagée par le mouvement historique, c'està-dire une phase transitoire où le pouvoir spirituel (les idées) et le pouvoir temporel (l'action politique, proprement dite) sont réunis dans les mains d'un seul ${ }^{99}$, et la perspective d'une pratique politique ${ }^{100}$. Il existe donc deux types de dictatures, les dictatures «progressives », dans le sens du

(94) «Pour Aulard le vrai moteur de la Révolution, ce ne sont pas les individus, mais l'ensemble des acteurs, depuis les plus larges masses jusqu'aux minorités actives, aux individus parfois indispensables, mais tirant leur force bien plus de leur groupe que d'eux-mêmes » (Claude NICOLET, L'idée républicaine en France..., op. cit., p. 99). Voir aussi Christian DELACROIX, François DossE et Patrick GARCIA, Les courants historiques en France, op. cit., p. 87.

(95) Voir Claude NICOLET, L'idée républicaine en France op cit, p. 101-105, plus récemment Claude NICOLET, La fabrique d'une nation. La France entre Rome et les Germains, Paris, Perrin, 2003 et Cristina CASSINA, « Comte devant la dictature », dans Michel BouRDEAU, Jean-François BraunsteIn et Annie PeTIT (dir.), Auguste Comte aujourd'hui, Paris, Kimé, 2003, p. 184-199.

(96) Claude NiCOLET, La fabrique d'une nation..., op. cit., p. 191.

(97) Regina PozZI, «Comte devant son siècle », dans Michel BourdeAu, Jean-François BRAUnSTEIN et Annie PETIT (dir.), op. cit., p. 137.

(98) Jean-François BRAUNSTEIN, «Comte "in context " : l'exemple de la sociologie », dans Michel BourdeaU, Jean-François BRAUnSTEIN et Annie PETIT (dir.), op. cit., p. 310.

(99) Cristina CASSINA, art. cit., p. 195-196.

(100) « La dictature n'est autre chose, au fond, que la concentration dans les mêmes mains, du pouvoir législatif et du pouvoir exécutif » (Docteur RoBINET, Notice sur la vie et l'ceuvre d'Auguste Comte, op. cit., p. 99). On retrouve ici dans des termes similaires le programme politique que Robinet prêtait à Turgot et, par extension, à Danton. 
mouvement historique, et les dictatures rétrogrades, qui vont contre lui ${ }^{101}$. Mais nous avons vu que le problème de la biographie de Danton, c'est qu'il est autant un militant politique qu'un homme d'État, déterminé par ses fonctions de pouvoir. En ce sens, même s'il est comparé aux grands dictateurs du positivisme, Louis XI, Henri IV ou Richelieu ${ }^{102}$, il reste une personnalité à part dans le système comtien.

Chez le docteur Robinet, Danton est appelé dictateur du moment où il devient ministre de la justice, fin août $1792^{103}$, jusqu'à son retrait du premier Comité de salut public, en juillet 1793, ce qui correspond, en gros, aux dix mois qu'Auguste Comte associe au règne de l'école de Diderot. Au cours de cette période riche en situations, deux faits se distinguent : l'organisation d'une défense militaire et l'instauration du gouvernement révolutionnaire. Sur ce premier point, Danton aurait été le seul à agir après le 10 août pour maintenir l'unité du gouvernement et repousser l'invasion ${ }^{104}$. Par ailleurs, sa nomination au Comité de salut public le 6 avril 1793 lui aurait permis d'appuyer la levée des 300000 hommes, dont Robinet lui attribue la paternitét ${ }^{105}$. Enfin, c'est en août qu'il demande la levée en masse ${ }^{106}$. Sur le second point, c'est d'abord par la lutte contre les girondins que s'inscrit la volonté d'établir un gouvernement révolutionnaire. Robinet écrit que « les girondins rêvaient de gouverner avec le régime anglais ${ }^{107}$ adapté à une forme politique plus ou moins républicaine $»^{108}$ et c'est l'impossibilité d'établir un tel système dans les circonstances de 1793 qui pousse à leur éviction ${ }^{109}$. Enfin, rappelant une formule d'Auguste Comte selon laquelle «il n'y a pas de société sans gouvernement », Robinet conclut que Danton aurait obtenu que « le comité de Salut public fût érigé en gouvernement $»^{110}$, ajoutant que l' « on est bien

(101) Claude NiCOLET, La Fabrique d'une nation..., p. 151. Robespierre serait l'archétype du dictateur anarchique, plaçant la métaphysique au dessus de l'affectif et de l'actif, quand Bonaparte serait un dictateur rétrograde, revitalisant le système théologico-monarchique que la Révolution a définitivement condamné. Entre les deux, la frontière est mince. Seul Danton incarne le type de dictature progressive (Alice GÉRARD, «La Révolution française d'Auguste Comte », art. cit., p. 79-82). Par ailleurs, le docteur Robinet avait pensé pendant un temps faire de Hoche le successeur de Danton (Docteur RoBINET, Le procès des Dantonistes, Paris, E. Leroux, 1879, p. 101).

(102) Docteur RoBINET, Danton Homme d'État, op. cit., p. 183.

(103) Ibidem, p. 104.

(104) Ibid., p. 105.

(105) Ibid., p. 134-137.

(106) Ibid., p. 194.

(107) Il faut comprendre un régime décentralisé

(108) Ibid., p. 176.

(109) Danton finit par se décider « enfin à profiter de son ascendant, alors général, et de sa présence au comité de Salut public, pour diriger et faire aboutir un mouvement [celui du 2 juin] qui, sans son acquiescement ou contre son gré, n'aurait pas réussi ou se serait égaré » (Ibid., p. 171).

(110) Ibid., p. 182. Il l'aurait demandé le $1^{\text {e }}$ août 1793. 
obligé de reconnaître [étant donné la situation d'alors] que la dictature du Comité de salut public fut aussi indispensable qu'inévitable, dut-elle verser dans le principal inconvénient de son institution : l'exagération de ses moyens et de sa durée ${ }^{111}$.

Ces deux éléments insistent sur le fait que le révolutionnaire aurait seul sauvé la France en 1793, et que c'est pour cette raison qu'il mérite d'être appelé « Homme d'État». L'œuvre politique dépasse en fait son créateur : ce qui sauve la France, c'est un gouvernement central et fort, capable d'imposer ses mesures aux provinces rebelles, par l'intermédiaire de ses commissaires, capable surtout d'organiser une défense nationale et la levée de troupes suffisamment nombreuses. Il paraît alors inconcevable que le dictateur Danton ait abandonné son poste, le 10 juillet, au profit de Robespierre, qui est élu le 27 juillet au Comité de salut public. D'après Robinet, il en fut contraint "pour fermer la bouche aux accusations d'ambition et d'aspiration à la dictature $»^{112}$. Par la suite, « il conserva sans doute une influence consultative sur la Convention; mais, placé hors du gouvernement qu'il avait créé, et auquel l'Assemblée se trouva elle-même bientôt étroitement subordonnée, il perdit rapidement, avec le pouvoir, tout ascendant moral $»^{113}$.

Ainsi, la dictature, chez Robinet, est avant tout une fonction politique qui sied aux personnages exceptionnels, mais elle est beaucoup plus attentive au contexte que chez Auguste Comte. Elle se présente comme un « empirisme transcendant ${ }^{114}$ qui surplombe certes le temps historique comme nous l'avons vu pour l'avant-gardisme supposé de Danton - mais ne le brusque pas. Elle a besoin, pour fonctionner, d'un appareil d'État que seul Danton a su établir, c'est-à-dire le gouvernement révolutionnaire. Si Robinet dénonce la mainmise de Robespierre sur le Comité de salut public, c'est parce que le dictateur ne correspond pas à ses attentes. Mais la forme de gouvernement, elle-même, ne le gêne absolument pas, signe évident de l'ambiguiité et de l'inadaptation à la IIIe République du programme de cette « dictature républicaine » demandée par les Positivistes orthodoxes, comme le souligne Claude Nicolet ${ }^{115}$.

(111) Ibid.

(112) Ibid., p. 191.

(113) Ibid., p. 194.

(114) Ibid., p. 182.

(115) Claude NiCOLET, L'idée républicaine en France..., op. cit., p. 240. Les Positivistes orthodoxes ont tout de même essayé d'actualiser le sens de cette expression. Robinet fait partie de ceux qui, avec Pierre Laffite, se sont le plus investis dans la politique, et qui s'opposent aux « maximalistes », plus autoritaires et anti-parlementaires comme le docteur Sémérie et Georges Audiffrant (voir Alice GÉRARD, « Les disciples « complets » d'Auguste Comte... », art. cit., p. 289). 
Alphonse Aulard n'aborde pas l'année 1793 dans les articles qu'il consacre à Danton, et il est difficile de comparer les deux analyses. Il parle cependant de la dictature lors de sa leçon d'ouverture, sans en questionner toute la portée épistémologique ${ }^{116}$. Sa lecture des événements est plus mesurée : Danton exercerait une «influence dominante au Conseil exécutif provisoire en août et en septembre 1792 », puis au premier Comité de salut public dont il est « le vrai chef, mais sans pouvoirs dictatoriaux, ne faisant qu'avec peine prévaloir ses avis », avant de se retirer de la politique car il est « dégoûté » ${ }^{117}$. Chez Aulard, il n'y a de dictature que par Robespierre et par Napoléon ${ }^{118}$. Cet angle mort de la polémique est assez étonnant, et montre assez bien comme l'ambiguïté du mot posait problème.

La controverse qui se joua lors de l'année 1893 entre l'interprétation positiviste et l'interprétation méthodique vit le triomphe de cette seconde aux dépens de la première. À la mort du docteur Robinet, en 1899, La Révolution française note qu'il « abonda trop dans son sens, il s'exagéra le rôle de Danton ${ }^{119}$ et regrette qu'il ait quitté la Société de l'histoire de la Révolution. Mais elle insiste sur le fait qu'il contribua à « saper la légende contre-révolutionnaire qui présentait Danton comme un assassin et un vendu $»^{120}$.

De fait, la vie privée de Danton ne fit l'objet d'aucune critique de la part d'Aulard, qui contribua même à renforcer la thèse de la probité du révolutionnaire ${ }^{121}$. Aulard ne déconstruisit pas entièrement le Danton des positivistes, et continua de promouvoir la mémoire de ce «bon Français avisé et agissant ${ }^{122}$, parce que cela servait à la formation d'une mémoire nationale. Par ailleurs, la question religieuse, centrale dans le système comtien, ne fit jamais l'objet d'une remarque de la part d'Alphonse Aulard, alors même qu'il consacra une partie de ses travaux à l'étude des cultes

(116) « La dictature ! On sait que pour Auguste Comte, la dictature est la condition du progrès jusqu'à l'avènement de la religion positive » (Alphonse AULARD, « Auguste Comte et la Révolution française », art. cit. , p. 26). Aulard lui reproche ensuite de ne pas nommer « le seul homme qui ait véritablement exercé dans la Révolution une sorte de dictature, Robespierre » (Ibid., p. 27).

(117) Ibid., p. 27-28.

(118) La question du coup d'État et de la dictature napoléonienne dans la pensée d'Aulard a été traitée par Pierre Serna (Pierre SERNA, «La République et le coup d'État. Les crises de la III République et la hantise du 18 Brumaire », Politix. Revue des sciences sociales du politique, vol. $10, \mathrm{n}^{\circ} 39,1997$, p. 131-154).

(119) $R F$, Tome XXXVII, $1899 / 2$, p. 472-473

(120) Ibidem, p. 472.

(121) Voir Alphonse Aulard, Études et leçons sur la Révolution française. Première série, Paris, F. Alcan, 1893, p. 137-183.

(122) Id., « Auguste Comte et la Révolution française », art. cit., p. 31. 
révolutionnaires ${ }^{123}$. Cela s'explique en partie parce qu'Aulard comme Robinet s'accordaient à voir en Danton l'exemple d'un homme politique laïc quoique le premier nuançât son influence à cet égard ${ }^{124}$.

La polémique fut donc avant tout celle de deux conceptions différentes de la République. Elle fut également celle de deux générations : Robinet, qui était né en 1825, avait connu plusieurs régimes politiques et subi très tôt l'influence de la pensée d'Auguste Comte ${ }^{125}$. Aulard, de son côté, faisait partie de la génération des historiens qui construisirent l'école méthodique en l'associant étroitement à la République comme horizon indépassable ${ }^{126}$. Ainsi, Danton pose - selon lui - le problème de l'interprétation historique et des discours qui l'alimentent, rappelant - également selon lui - que l'histoire n'est jamais totalement libre, et que ce parti pris en fait sa richesse. L'historien dans la République reste un historien «en son temps », et Michel Vovelle a rappelé combien le poste d'Aulard à la Sorbonne pouvait être considéré comme un «poste de combat $»^{127}$. La frontière qui sépare ces deux historiens dantonistes est celle de la méthode et du lieu d'où l'on parle : la rue Monsieur le Prince ou la Sorbonne. Elle valide le soutien « officiel » de la parole aulardienne.

De même, la lecture de la controverse Aulard / Robinet peut se faire à l'aune de l'opposition entre sociologie et histoire qui marque la fin du $\mathrm{XIX}^{\mathrm{e}}$ siècle et le début du $\mathrm{XX}^{\mathrm{e}}$ siècle. La critique aulardienne des thèses comtienne est finalement très proche de la réponse donnée aux sociologues par Charles Seignobos dans La méthode historique appliquée aux sciences sociales : elle nuance l'interprétation des faits historiques par des systèmes généralisants et englobants, pour privilégier au sein de l'histoire la place

(123) Id., Le culte de la Raison et le culte de l'être suprême, Paris, Felix Alcan, 1892 ; La Révolution française et les congrégations, Paris, E. Cornély, 1903 ; Le Christianisme et la Révolution française, Paris, Rieder, 1925.

(124) Docteur RoBINET, Le mouvement religieux à Paris pendant la Révolution (1789-1801). Tome II - Préliminaires de la déchristianisation, 1898, p. 3; Alphonse AULARD, « Le culte de l'Être suprême. La réaction contre le culte de la Raison », $R F$, Tome XXI, 1891/2, p. 10-11 et « Le culte de la Raison. Les préliminaires », $R F$, Tome XX, 1891/1, p. 112. L'analyse des cultes de la Raison et de l'Être suprême par Aulard fait systématiquement appel aux philosophes, qu'ils fussent Diderot, Voltaire (pour les Hébertistes) ou Rousseau (pour les Robespierristes). Il semble que ce soit par l'étude de ces cultes qu'Alphonse Aulard ait trouvé les arguments suffisants pour déconstruire le système comtien lors de sa leçon de décembre 1892. cit., p. 7)

(125) Selon Émile Corra, il aurait été attiré par le positivisme dès 1848 (Émile CORRA, op.

(126) Voir Pim DEN BoER, History as a profession : the study of history in France, 1818-1914, Princeton, Princeton University Press, 1998 et Patrick GARCIA, « Historiographie méthodique », art. cit., p. 448

(127) Michel VovelLe, «La galerie des ancêtres », dans Combats pour la Révolution française, op. cit., p. 14. 
de l'individu conscient ${ }^{128}$. Ainsi, même si la « sociologie » défendue par Robinet est très différente de celle des disciples de Durkheim, elle montre qu'aux deux extrémités du «moment méthodique », le dialogue entre histoire, philosophie et sociologie fut constant.

Cette manière d'utiliser la controverse pour construire son appareil scientifique préfigure la venue du jeune Mathiez qui, reprenant Danton à son compte, utilisera les preuves de sa corruption pour s'opposer à son maître ${ }^{129}$. Il semble toutefois que cette reprise tardive se soit trouvée en terrain conquis ${ }^{130}$. De là l'absence d'une vraie polémique de fond, dont on a souvent l'impression que Mathiez l'alimenta seul, lors même qu'il fut l'un des premiers historiens à s'intéresser aux thèses d'Émile Durkheim ${ }^{131}$. Face à l'élève hérétique, le maître ne fut guère expansif, se contentant de republier un élogieux article - paru quelques années auparavant ${ }^{132}$ - pour dissocier deux Mathiez :

«M. A. Mathiez, écrivit-il, par une aventure étrange, a un sosie, ou plutôt un homonyme (même nom de famille, même prénom), qui écrit dans un nouveau périodique, les Annales révolutionnaires, et qui s'applique avec soin à prendre le contrepied des amitiés et de la méthode de notre excellent collaborateur et ami $»^{133}$.

On appréciera le procédé.

Alric MABIRE

Université de Rouen alric.mabire@gmail.com

(128) Christian Delacroix, François DosSE et Patrick Garcia, Les courants historiques en France, op. cit., p. 108-109.

(129) Voir James FrIGUGLIETTI, Albert Mathiez, historien révolutionnaire (1874-1932), op. cit. et « La querelle Mathiez-Aulard et les origines de la Société des études robespierristes », art. cit.

(130) L'étude des tables de La Révolution française nous révèle que si le nombre d'articles consacrés à Danton est de douze pour la période 1890-1895 (Tomes XIX à XXVIII), il est de quatre pour 1895-1902 (Tomes XXIX à XLIII), de six pour 1903-1910 (Tomes XLIV à LIX) et de un pour 1911-1914 (Tomes LX à LXVII). Cette absence relative du révolutionnaire marque la tiédeur du « dantonisme » d'Aulard (voir Claude NICOLET, L'idée républicaine en France, op. cit., p. 97).

(131) James Friguglietti a rappelé le débat houleux qui accompagna la soutenance de thèse du jeune Mathiez à propos des cultes révolutionnaires. Mathiez avait repris à Durkheim ses théories sur les phénomènes religieux comme déterminés par leurs formes (croyances et pratiques collectives), ce qui occasionna une dispute entre le jury et le thésard (James FrigugLiETTI, « La querelle Mathiez-Aulard et les origines de la Société des études robespierristes », art. cit.).

(132) Albert MATHIEZ, « M. Aulard, historien et professeur », $R F$, Tome LV, 1908/2, p. 46-60. (133) $R F$, Tome LV, 1908/2, p. 81 . 